\title{
Proton Zemach radius from measurements of the hyperfine splitting of hydrogen and muonic hydrogen
}

\author{
Arnaud Dupays, Alberto Beswick, Bruno Lepetit, and Carlo Rizzo \\ LCAR-IRSAMC, Université Paul Sabatier, \\ 118 Route de Narbonne, 31062 Toulouse cedex 04, France \\ Dimitar Bakalov \\ Institute for Nuclear Research and Nuclear Energy, \\ Tsarigradsko chaussée 72, Sofia 1784, Bulgaria
}

\begin{abstract}
While measurements of the hyperfine structure of hydrogen-like atoms are traditionally regarded as test of bound-state QED, we assume that theoretical QED predictions are accurate and discuss the information about the electromagnetic structure of protons that could be extracted from the experimental values of the ground state hyperfine splitting in hydrogen and muonic hydrogen. Using recent theoretical results on the proton polarizability effects and the experimental hydrogen hyperfine splitting we obtain for the Zemach radius of the proton the value $1.040(16) \mathrm{fm}$. We compare it to the various theoretical estimates the uncertainty of which is shown to be larger that $0.016 \mathrm{fm}$. This point of view gives quite convincing arguments in support of projects to measure the hyperfine splitting of muonic hydrogen.
\end{abstract}




\section{INTRODUCTION}

The hyperfine splitting of the ground state of the hydrogen atom is among the most accurately measured quantities [1, 2]:

$\Delta E_{\text {exp }}^{\mathrm{HFS}}=1420405751.7667 \pm 0.0009 \mathrm{~Hz}$

The relative experimental uncertainty in Eq. 1 does not exceed $10^{-12}$. The theoretical predictions for $\Delta E^{\mathrm{HFS}}$, based on QED, are less accurate. This is partly due to the computational difficulties which increase very fast for the higher order terms in the perturbative expansion in powers of $\alpha$ and $(Z \alpha)$ [3, 4], and to the limited precision of the fundamental constants involved (the Rydberg known to $10^{-11}$, electron to proton mass ratio and $\alpha$ known to parts of $10^{-8}[3]$ ). The main uncertainties come, however, from the insufficient knowledge of the structure of protons. Because of this, the comparison of theoretical results with the experimental value of Eq. 1 can not test QED beyond the contribution of proton polarizability effects of the order of a few ppm. To perform a more precise test of QED, therefore, either additional information on the electromagnetic structure of protons should be used, or the comparison should be done between theoretical and experimental results on the hyperfine splitting in hydrogen-like bound states of charged point-like leptons. Muonium is most appropriate for the latter; indeed, the recent measurements of the hyperfine splitting of the ground state of muonium [5] have been shown to agree with theory up to $0.510^{-7}$ (see [3] and references therein), so that the correctness of QED results about the hyperfine splitting of hydrogen-like atoms has been experimentally confirmed at least with the same precision. As for the use of information on the electromagnetic structure of protons from other sources in attempts to reduce the theoretical uncertainty of $\Delta E^{\mathrm{HFS}}$, until recently there was no theoretical progress in this direction, and the only realistic idea for years seemed to be to estimate the proton structure contributions from complementary measurements of the hyperfine splitting in muonic hydrogen [6, 7]. We are now going to critically analyze this idea in the context of recent theoretical results on the polarizability of protons [8, 9], of the development of new precision spectroscopy instrumentation [10], and of our better understanding of the dominating proton structure contributions to the hyperfine splitting in hydrogen-like atoms. This will lead us to the alternative point of view to look at the hyperfine splitting measurements in hydrogen and muonic hydrogen as measurements of the Zemach radius of the proton by assuming that all QED predictions are credible. This way 
we shall obtain a "first experimental" value of the proton Zemach radius from the hyperfine splitting of hydrogen which may be compared to theoretical values based on different proton form factor fits.

\section{HYPERFINE SPLITTING OF HYDROGEN ATOM GROUND STATE}

To analyze the various sources of uncertainty in the theoretical value of $\Delta E^{\mathrm{HFS}}$ we put it in the traditional form [1]]:

$\Delta E_{t h}^{\mathrm{HFS}}=E^{\mathrm{F}}\left(1+\delta^{\mathrm{QED}}+\delta^{\mathrm{str}}\right)$

where $E^{\mathrm{F}}$ is the Fermi splitting [12] expressed in terms of the electron and proton masses $m_{e}, m_{p}$ and the dipole magnetic moment of the proton $\mu_{p}$ :

$E^{\mathrm{F}}=\frac{8}{3} \alpha^{4} c^{2} \frac{m_{e}^{2} m_{p}^{2}}{\left(m_{e}+m_{p}\right)^{3}} \mu_{p}$

while $\delta^{\mathrm{QED}}$ and $\delta^{\text {str }}$ are correction terms related to higher order QED effects and to proton electromagnetic structure due to strong interactions. (Eq. 2 is only correct in the leading order, since higher orders QED and structure effects mix up 13|.) Up to terms of order $O\left(\alpha^{3}\right)$ (without distinguishing $\alpha$ from $\left.(Z \alpha)\right), \delta^{\mathrm{QED}}$ is given by [3], 4]]:

$\delta^{\mathrm{QED}}=a_{e}+\frac{3}{2} \alpha^{2}+\alpha^{2}\left(\log 2-\frac{5}{2}\right)-\frac{8 \alpha^{3}}{3 \pi} \log \alpha\left(\log \alpha-\log 4+\frac{281}{480}\right)+18.984 \times \frac{\alpha^{3}}{\pi}+\ldots$

where $a_{e}$ is the anomalous magnetic moment of the electron. Note that the expression for $\delta^{\text {QED }}$ does not involve the mass ratio $m_{e} / m_{p}$; all terms which depend on proton mass or come from strong interactions are included in $\delta^{\text {str }}$. In turn, $\delta^{\text {str }}$ splits into a "static" part $\delta^{\text {rigid }}$ that accounts for the elastic electromagnetic form factors of the proton and can be calculated using data from elastic scattering experiments, a part $\delta^{\text {pol }}$ that comes from the internal dynamics of the proton and could only be evaluated using data on inelastic processes with protons, and a part $\delta^{\text {hvp }}$ describing the strong interaction effects outside the proton, such as hadron vacuum polarization: $\delta^{\text {str }}=\delta^{\text {rigid }}+\delta^{\text {pol }}+\delta^{\text {hvp }}$. Two types of "static" proton structure corrections are incorporated in $\delta^{\text {rigid }}$, associated with the spatial distribution of the charge and magnetic moment within the proton and with recoil effects, respectively: $\delta^{\text {rigid }}=\delta^{\text {Zemach }}+\delta^{\text {recoil }}$. The former has been calculated by Zemach [14] and may be put in the form [8]:

$\delta^{\text {Zemach }}=\frac{2 \alpha m_{e p}}{\pi^{2}} \int \frac{d^{3} p}{\left(\mathbf{p}^{2}+\alpha^{2} m_{e p}^{2}\right)^{2}}\left(\frac{1}{\mu_{p}} G_{E}\left(-\mathbf{p}^{2}\right) G_{M}\left(-\mathbf{p}^{2}\right)-1\right)=-2 \alpha m_{e p} R_{p}+O\left(\alpha^{3}\right)$ 
where $m_{e p}=m_{e} m_{p} /\left(m_{e}+m_{p}\right)$ and $R_{p}$ is the first moment of the convolution of the proton charge and magnetic moment distributions, also known as Zemach radius of the proton. The recoil correction $\delta^{\text {recoil }}$ denotes the contribution of all terms which depend on the ratio $m_{e} / m_{p}$; for sake of simplicity we skip here the rather lengthy explicit expression of $\delta^{\text {recoil }}$ which may be found in [11]. The proton polarizability correction $\delta^{\mathrm{pol}}$ and the hadron vacuum polarization correction have been evaluated recently [9, 15], using the available data on the proton polarized structure functions and on electron-positron annihilation into hadrons.

Eq. 2] may now be put in a more detailed form:

$\Delta E_{t h}^{\mathrm{HFS}}=E^{\mathrm{F}}\left(1+\delta^{\mathrm{QED}}+\delta^{\mathrm{Zemach}}+\delta^{\text {recoil }}+\delta^{\mathrm{pol}}+\delta^{\mathrm{hvp}}\right)$

It is important for what follows to review the order of magnitude of the various terms in the right-hand side of Eq. 6 and briefly discuss the uncertainty of each of them. The uncertainty of $E^{\mathrm{F}}$ is due to the uncertainty of $m_{e}, m_{p}, \mu_{p}$ and $\alpha$ and does not exceed $0.01 \mathrm{ppm}[16]$. $\delta^{\text {QED }}$ is dominated by the anomalous magnetic moment of the electron; the uncertainty of the term comes from the uncalculated terms of relative order $O\left(\alpha^{4}\right)$ and higher and is estimated not to exceed $0.001 \mathrm{ppm} \mathrm{[3].} \delta^{\text {rigid }}$ is close to $40 \mathrm{ppm}$ (11] and references therein); its uncertainty - 2-3 ppm - is due to the uncertainty of the actual values of the proton radii and is of the order of $5 \%$. The recoil term $\delta^{\text {recoil }} \approx 5.68 \mathrm{ppm}$ [4] adds little uncertainty to $\delta^{\text {rigid }}$. As for the proton polarizability correction $\delta^{\text {pol }}$, until recently there existed only the upper limit $\delta^{\mathrm{pol}}<4 \mathrm{ppm}$ [4], obtained by ascribing the whole discrepancy between $\Delta E_{e x p}^{\mathrm{HFS}}$ and $\Delta E_{t h}^{\mathrm{HFS}}$ to the contribution from $\delta^{\mathrm{pol}}$. The present value of the proton polarizability correction, calculated on the ground of experimental data on the polarized structure function [9], is $1.6 \pm 0.6 \mathrm{ppm}$. The hadron vacuum polarization correction $\delta^{\text {hvp }} \sim 10^{-8}[17]$ is much too small. The overall uncertainty of $\Delta E_{t h}^{\mathrm{HFS}}$ is therefore of the order of 2-3 ppm and is entirely due to proton structure effects. All discussed quantities are summarized in the leftmost two columns of Table III the righmost two columns of the Table contain the numerical values of the corresponding quantities for muonic hydrogen and will be discussed in next section.

As already pointed out, the challenging comparison of $\Delta E_{t h}^{\mathrm{HFS}}$ and $\Delta E_{e x p}^{\mathrm{HFS}}$ for hydrogen can not be regarded as a test of the QED contributions $\delta^{\mathrm{QED}}$ of order $O\left(\alpha^{3}\right)$ and higher because of the significant overall theoretical uncertainty. We adopt instead an alternative point of view to assume that the theoretical values of $\delta^{\mathrm{QED}}, \delta^{\text {recoil }}, \delta^{\text {hvp }}$ and $\delta^{\text {pol }}$ are accurate 
TABLE I: Magnitude and uncertainty of the contributions to the hyperfine splitting of the ground state of hydrogen and muonic hydrogen from various correction terms.

\begin{tabular}{|l|rr|rr|}
\cline { 2 - 5 } \multicolumn{1}{c|}{} & \multicolumn{2}{c|}{ Hydrogen } & \multicolumn{2}{c|}{ Muonic Hydrogen } \\
\cline { 2 - 5 } & magnitude & uncertainty & magnitude & uncertainty \\
\hline$E^{\mathrm{F}}$ & $1420 \mathrm{MHz}$ & $0.01 \mathrm{ppm}$ & $182.443 \mathrm{meV}$ & $0.1 \mathrm{ppm}$ \\
\hline \hline$\delta^{\mathrm{QED}}$ & $1.1610^{-3}$ & $<0.00110^{-6}$ & $1.1610^{-3}$ & $10^{-6}$ \\
$\delta^{\text {rigid }}$ & $3910^{-6}$ & $210^{-6}$ & $7.510^{-3}$ & $0.110^{-3}$ \\
$\delta^{\text {recoil }}$ & $610^{-6}$ & $10^{-8}$ & $1,710^{-3}$ & $10^{-6}$ \\
$\delta^{\text {pol }}$ & $1.410^{-6}$ & $0.610^{-6}$ & $0.4610^{-3}$ & $0.0810^{-3}$ \\
$\delta^{\text {hvp }}$ & $10^{-8}$ & $10^{-9}$ & $0.0210^{-3}$ & $0.00210^{-3}$ \\
\hline
\end{tabular}

and use the experimental data to determine the Zemach radius of the proton $R_{p}$ as:

$R_{p}=-\left(\Delta E_{\text {exp }}^{\mathrm{HFS}} / E^{\mathrm{F}}-1-\delta^{\mathrm{QED}}-\delta^{\mathrm{recoil}}-\delta^{\mathrm{pol}}-\delta^{\mathrm{hvp}}\right) /\left(2 m_{e p} \alpha\right)$

The above assumption is justifiable since all four correction terms are objects of QED, the only difference of $\delta^{\text {hvp }}$ and $\delta^{\text {pol }}$ from the former two being that their evaluation requires the use of additional phenomenological information beyond first principles. From Eq. [7] we get the experimental value $R_{p}=1.040(16) \mathrm{fm}$, where the uncertainty $\pm 0.016 \mathrm{fm}$ comes from the theoretical uncertainty of $\delta^{\text {pol }}[9]$.

The Zemach radius of the proton is defined in terms of an integral of the charge and magnetic form factors of the proton $G_{E}(k)$ and $G_{E}(k)$ over space-like transfer momenta $k, k^{2}=-\mathbf{k}^{2}$ (see Eq. 5 , or equivalently, by the first moment of the convolution of the charge and magnetic moment distributions $\rho_{E}(r)$ and $\rho_{M}(r)$ in coordinate space [14]:

$R_{p}=\int d^{3} r r \int d^{3} r^{\prime} \rho_{E}\left(\mathbf{r}-\mathbf{r}^{\prime}\right) \rho_{M}\left(\mathbf{r}^{\prime}\right)$

The directly observable quantity which is most sensitive to the Zemach radius of the proton is the hyperfine splitting of bound systems involving protons (compare to the Lamb shift which is related to the proton r.m.s. charge radius [18].) The experimental value of $R_{p}$ sets important restrictions on the theoretical models of proton electromagnetic structure and, in particular, on the parametrization of proton form factors, in terms of which the theoretical values are calculated. Consider as an example the values of $R_{p}$ calculated from Eq. 5 using a 
few popular approximations of the proton form factors. Numerical calculations give $R_{p}=1.02$ fm for the dipole fit, and $R_{p}=1.067 \mathrm{fm}$ for the fit of [19]; unfortunately, no information on the uncertainty of the parameters of the fit is available, and no conclusions could be made on the compatibility of these values with the "experimental" one. Both fits are consistent with $\mu_{p} G_{E}\left(-\mathbf{k}^{2}\right) / G_{M}\left(-\mathbf{k}^{2}\right) \approx 1$ for $\mathbf{k}^{2}<5 \mathrm{GeV}^{2} / \mathrm{c}^{2}$. To account for the recent experimental results on the form factor ratio [20], we also evaluated $R_{p}$ by using the Simon's fit for either the charge or magnetic form factor and expressing the other one using the relation $\mu_{p} G_{E}\left(-\mathbf{k}^{2}\right) / G_{M}\left(-\mathbf{k}^{2}\right)=1-0.13\left(\mathbf{k}^{2}-0.04\right)$ [20], and got $R_{p}=1.060 \mathrm{fm}$ and $R_{p}=1.073$ fm respectively. Though preliminary, these estimates show that the current theoretical uncertainty of $R_{p}$ significantly exceeds the experimental one, and that the experimental results on the proton Zemach radius may be used as a test for the quality of models of the proton in the limit of low transfer momenta.

\section{HYPERFINE SPLITTING OF MUONIC HYDROGEN ATOM GROUND STATE}

Muonic hydrogen is the only other hydrogen-like atom in which the hyperfine splitting of the ground state could be measured with high precision. Due to the large muon mass $m_{\mu} / m_{e} \approx 210^{2}$, the binding energy of the ground state of muonic hydrogen is of the order of $200 \mathrm{Ry}$, and the radius of the muon orbit is $\sim a_{0} / 200$ so that the energy levels of muonic hydrogen are more "sensitive" to the details of the proton structure than the levels of normal hydrogen. The expressions in Eqs. 23 and 5 for the hyperfine splitting of a hydrogen-like atom and the various contributions to it apply for muonic hydrogen as well; however, the energy scale and the relative size of the various terms differ significantly from the hydrogen case (see the rightmost two columns of Table II) The Fermi splitting now is 183 meV that corresponds to a hyperfine transition wavelength of $6.1 \mu \mathrm{m}$. The explicit form of the higher order terms in $\delta^{\text {QED }}$ may differ from Eq. 4 since different momenta are expected to give the main contribution in loop integrations, while proton structure modifies the proton voerteces. We do not know of any published explicit expression of $\delta^{\mathrm{QED}}$ for the ground state of muonic hydrogen. To our opinion the lack of interest in the topic is due to the unclear perspectives of an experimental verification of the theoretical results. Indeed, the analogous contributions to the Lamb shift of muonic hydrogen were evaluated with a very high accuracy [21, 22] as 
soon as the proposal for the experiment [23] was about to be put forward. We are therefore convinced that the uncertainty of $\delta^{\mathrm{QED}}$ may be brought down to $0.1 \mathrm{ppm}$ if necessary. The evaluation of the recoil terms $\delta^{\text {recoil }}$ may not be that easy since the mass ratio $m_{\mu} / m_{p} \sim 0.11$ is much larger compared to hydrogen, and terms of order $O\left(\alpha\left(m_{\mu} / m_{p}\right)^{n}\right), n=2,3,4$, are all expected to contribute by more than $1 \mathrm{ppm}$. Most appropriate in this case might be an essentially two-body approach based on the quasipotential equation [24, 25]; we assume that the theoretical uncertainty may be brought down below $10^{-6}$ this way, and put $1 \mathrm{ppm}$ in the rightmost column of Table [1] Since we do not know of any published result on $\delta^{\text {recoil }}$, we take as estimate of the magnitude of the recoil effects the formula for the leading recoil term in muonium of Ref. [4] and get $\delta^{\text {recoil }} \sim-(3 \alpha / \pi)\left(m_{\mu} / m_{p}\right) \ln \left(m_{\mu} / m_{p}\right) \sim 1.710^{-3}$. The proton polarizability correction has been evaluated using the same methods as for hydrogen: $\delta^{\text {pol }}=(4.6 \pm 0.8) \times 10^{-4}[8]$. The term $\delta^{\text {hvp }}$ describing the hadron vacuum polarization was shown to contribute by approximately 20 ppm [26], unlike hydrogen where it does not give any considerable contribution in hydrogen.

With the content of Table $\llbracket$ in mind, we are now ready to discuss the information that would be provided by measurements of the hyperfine splitting of the ground state of muonic hydrogen atoms.

The proton structure correction $\delta^{\text {str }}$ in muonic hydrogen is enhanced (compared to hydrogen) by a factor of $210^{2}$. Therefore, a measurement of $\Delta E^{\mathrm{HFS}}$ in $\left(\mu^{-} p\right)_{1 s}$ can not be a good test of QED since QED effects are overshadowed by the proton structure corrections. Further on, in both hydrogen and muonic hydrogen, the proton structure corrections $\delta^{\text {str }}$ is dominated by two independent terms: the Zemach term $\delta^{\text {rigid }}$ and the polarizability term $\delta^{\mathrm{pol}}$. While the Zemach term is directly related to a well defined physical parameter - the Zemach radius of the proton $R_{p}$ (see Eq. [5),$\delta^{\text {pol }}$ is expressed in terms of the form factors and polarized structure functions of the proton in an indirect and case-dependent way and is not associated with a single parameter. Compared to hydrogen, both these terms scale approximately as $\left(m_{\mu} / m_{e}\right)$. This all brings us to the conclusion that opposite to what was believed by some authors $\left[6\right.$, 7], the measurements of $\Delta E^{\mathrm{HFS}}$ in hydrogen and muonic hydrogen atoms are not complementary in a sense which would let us extract the values of two universal parameters of the proton, characterizing its charge and magnetic distribution and polarizability. However, if assuming that all terms in the right-hand side of Eq. 7 are evaluated correctly by theory, these measurements may be regarded as repeated experimen- 
tal determination of the Zemach radius of the proton. While the discutible point in this assumption is the credibility ot the theoretical evaluation of $\delta^{\text {pol }}$ (if neglecting $\delta^{\text {hvp }}$ ), the repeated measurements of $R_{p}$ in hydrogen and muonic hydrogen are the best way to verify it: compatible values of $R_{p}$ extracted from the hyperfine splitting in hydrogen and muonic hydrogen will confirm the reliability of the theoretical values of $\delta^{\mathrm{pol}}$ and vice versa.

The accuracy of $R_{p}$ depends on the uncertainty of $\delta^{\text {pol }}$; a measurement of the hyperfine splitting of the ground state of muonic hydrogen based on the results of [8] would give the value of $R_{p}$ accurate to $1 \%$. This would be more precise than the value obtained in the previous section because of the smaller relative uncertainty of the theoretical uncertainty of $\delta^{\mathrm{pol}}$ (see Table II), however, things may change with the more refined theoretical results to come in the future. As already mentioned, such an accuracy would fairly allow to filter the numerous theoretical estimates of $R_{p}$ and detect a deviation of $G_{E} / G_{M}$ from 1 by distinguishing the values of $R_{p}$ obtained with and without account of the JLab experimental results [20]. It would be preferable for this purpose to have the value of $R_{p}$ accurate to $0.5 \%$ or better, that requires in turn that the theoretical uncertainty of $\delta^{\text {pol }}$ be brought below $310^{-5}$ and that the experimental error of $\Delta E_{\text {exp }}^{\mathrm{HFS}}$ not exceed $30 \mathrm{ppm}$.

The muonic hydrogen Lamb shift experiment, currently in progress at PSI [23], may provide at a later stage as a by-product the hyperfine splitting of the $2 S$-state of the muonic hydrogen atom with a relative accuracy of the order of $0.510^{-3}$. According to [21], the Zemach correction $\delta^{\text {rigid }}$ for the $2 S$-state is again $\sim 810^{-3}$. Unless the (yet uncalculated) polarization correction $\delta^{\text {pol }}$ happens to be anomalously large in this case, this measurement would therefore provide the value of $R_{p}$ with an accuracy of $5-10 \%$ [23] which is below the expected accuracy of the measurements in the $1 \mathrm{~S}$ state. The significant improvement of the accuracy of the proton rms charge radius expected from PSI experiment will not help imcreasing the accuracy of $R_{p}$ either because $R_{p}$ is independent of the proton rms charge radius. In order to determine the Zemach radius of the proton from the hyperfine splitting of muonic hydrogen atoms in the $1 s$ or $2 s$ states with accuracy $1 \%$ or better, the experimental error of $\Delta E_{\text {exp }}^{\mathrm{HFS}}$ should not exceed $50 \mathrm{ppm}$ - a requirement that is not met by the PSI Lamb shift experiment 23]. An alternative experimental method, based on the response of the muon transfer rate from hydrogen to oxygen to the population of the $\left(\mu^{-} p\right)_{1 S}$ para-state 27], was proposed recently [28]. The method takes advantage of the recent progress in the development of tunable lasers in the far infrared range around $6.1 \mu \mathrm{m}[10]$. The efficiency of 
the method has been demonstrated by means of Monte Carlo simulations. The experimental error limits have not been discussed; the main source of experimental uncertainty is expected to be the Doppler broadening of the transition lines.

\section{HYPERFINE SPLITTING OF MUONIC HYDROGEN MOLECULAR ION}

In conclusion, we would like to briefly outline an alternative possibility for determining the proton Zemach radius from a measurement of the hyperfine splitting of the muonic molecular ion $p \mu^{-} p$. The hydrogen muonic molecular ions $\left(p \mu^{-} p\right)$ are formed in the excited ortho-state with orbital momentum $J=1$ in collisions of muonic hydrogen atoms with hydrogen molecules 29]:

$\left(\mu^{-} p\right)+H_{2} \rightarrow\left[(p \mu p)_{J} p e e\right]^{*}$

The formation rate $\lambda_{p u p}$ is proportional to the hydrogen target number density $\varphi: \lambda_{p \mu p}=$ $\varphi / \varphi_{0} \times 2.210^{6} \mathrm{~s}^{-1}[30]$. At high densities $\lambda_{p \mu p}$ exceeds the muon weak decay rate $\lambda_{0}=$ $0.4510^{6} \mathrm{~s}^{-1}$ so that the muons spend most of their life time bound in muonic molecular ions. The spin-orbit and spin-spin interactions of the protons and the muon split the ortho-state with $J=1$ into 5 hyperfine state labeled with the quantun numbers $(s F)$ of the total spin $\mathbf{s}=\mathbf{s}_{p 1}+\mathbf{s}_{p 2}+\mathbf{s}_{\mu}$ and the total angular momentum $\mathbf{F}=\mathbf{s}+\mathbf{J}[30,31]$; the para-state $J=0$ has no hyperfine structure. The energy separation between the ortho-levels with $s=1 / 2$ and $s=3 / 2$ is $\sim 138 \mathrm{meV}$, while the separation within each of these groups is two orders of magnitude smaller.

A measurement of the splitting between the hyperfine states with $s=1 / 2$ and $s=3 / 2$ might be based on the strong dependence of the rate of spontaneous ortho-para transitions $\lambda_{o p}=\lambda_{o p}(s)$ on $s: \lambda_{o p}(1 / 2)=7.210^{4} \mathrm{~s}^{-1}, \lambda_{o p}(3 / 2)=0.810^{4} \mathrm{~s}^{-1}$. Since only the ortho-states with $s=1 / 2$ are initially populated at high densities, the observable ortho-para transition rate is close to $\lambda_{o p}(1 / 2)$. The idea of the experimental method would be to use a tunable laser to stimulate transitions from the $s=1 / 2$ to the $s=3 / 2$ hyperfine states, for which $\lambda_{o p}(3 / 2)$ is an order of magnitude smaller, which would result in a resonance drop of the observable rate of ortho-para transitions. Though such an experiment would require the development of tunable far IR narrow band lasers in the range of $8.8 \mu \mathrm{m}$, it has some significant advantages compared with measurement in gaseous hydrogen at room tempertures - suppressed Doppler 
broadening, small muon stopping volume, high energy density of the laser beam, high rate of laser- stimulated ortho-para transitions - which deserve a careful consideration in future. As for the theory, the accuracy of the currently available results on the hyperfine structure of $\left(p \mu^{-} p\right)_{J=1}$ is limited to the leading order Breit and Zemach corrections and to the muon anomalous magnetic moment [31, 32]; therefore, the theoretical uncertainty is currently about $10^{-4}$. In order to determine $R_{p}$ to $1 \%$ from $p \mu^{-} p$ hyperfine splitting measurements, next order QED effects have to be taken into account together with proton polarizability and molecular ion finite size effects [33, 34] so that the theoretical uncertainty be reduced by an order of magnitude to meet an experimental error below $50 \mathrm{ppm}$. This is a challenging task since $\left(p \mu^{-} p\right)$ is a bound system of particles with comparable masses in which three-body relativistic dynamics may show up yet in the next-to-leading order.

\section{CONCLUSIONS}

By assuming that the numerical results for the various terms in the theoretical expression for the hyperfine splitting of the ground state of the hydrogen atom are correct (within the limits of the claimed accuracy), we have determined the value of the proton Zemach radius $R_{p}$. We have also demonstrated that comparison of the experimental value of $R_{p}$ with theoretical calculation is a sensitive test of the quality of the approximation of the proton form factors at low momentum transfer and of the possible deviation of the ratio of the charge and magnetic form factors from 1. To verify the credibility of the theoretical evaluation of proton polarizability effects, we consider the possibility of measuring the hyperfine splitting of the ground state of muonic hydrogen atoms or, in a remote perspective, of muonic hydrogen molecular ions.

\section{ACKNOWLEDGMENT}

The work on the paper was supported by NATO collaborative linkage grant PST.CLG.978454. D.B. also recognizes the partial support of grant Phy-1001 of the Bulgarian National Fund for Scientific Research. The authors are grateful to A.P. Martynenko 
and A. Le Padellec for helpful discussions.

[1] H. Hellwig, R. F. C. Vessot, M. V. Levine, et al., IEEE Trans. Instrum. Meas. IM-19, 200 (1970).

[2] L. Essen, M. J. Donaldson, M. J. Bangham, and E. G. Hope, Nature 229, 110 (1971).

[3] M. I. Eides, H. Grotch, and V. A. Shelyuto, Phys. Reports 342, 63 (2001).

[4] J. R. Sapirstein, and D. R. Yennie, in Quantum Electrodynamics, Ed. T.Kinoshita, (World Scientific, 1991), p.560.

[5] W. Liu, M. G. Boshier, and S. Dhawan, Phys. Rev. Lett. 82, 711 (1999).

[6] Bakalov, D. et al., Phys. Lett. A 172, 277, (1992).

[7] Bakalov, D. et al., in Proceedings of the III International Symposiun "WEIN-92", Dubna, Ed. Ts.D. Vylov, (World Sci., 1992), p. 656.

[8] E. V. Cherednikova, R. N. Faustov, and A. P. Martynenko, Nucl. Phys. A 703, 365 (2002),

[9] R. N. Faustov, A. P. Martynenko, and V. A. Saleev, Physics of Atomic Nuclei 62, 2099 (1999).

[10] F. Kottmann, F. Biraben, C. A. N. Conde, et al., in Quantum electrodynamics and physics of the vacuum, Ed. G.Cantatore, AIP Conference proceedings 564, 13 (2001).

[11] G. T. Bodwin, and D. R. Yennie, Phys. Rev. D 37, 498 (1988).

[12] E. Fermi, Z. Phys. 60, 320 (1930).

[13] S. G. Karshenboim, Phys. Lett. A 225, 97 (1997).

[14] A. C. Zemach, Phys. Rev. 104, 1771 (1956).

[15] Faustov, R.N., Martynenko, A.P., Eur. Phys. J. C 24, 281 (2002).

[16] K. Hagiwara, et al. Phys. Rev. D 66, 01001, (2002)

[17] R. N. Faustov, A. Karimkhodzhaev, and A. P. Martynenko, Yadernaya fizika 62, 2284 (1999).

[18] K. Pachucki, Phys. Rev. A 60, 3593 (1999).

[19] G. G. Simon, C. Schmitt, F. Borkowski, and V. H. Walther, Nucl. Phys. A 333, 381 (1980).

[20] O. Gayou, et al., Phys. Rev. Lett. 88, 092301 (2002).

[21] K. Pachucki, Phys. Rev. A 53, 2092 (1996).

[22] T. Kinoshita, and M. Nio, Phys. Rev. Lett. 82, 3240 (1999).

[23] P. Hauser, C. Petitjean, L. M. Simons, D. Taqqu, F. Kottmann, et al., Proposal for an 
experiment at PSI, R-98-03.1, (1999).

[24] I. T. Todorov, Phys. Rev. D 3, 2351 (1971).

[25] V. V. Dvoeglazov, R. N. Faustov, and Yu. N. Tukhtyaev, Part. Nucl. 25, 58 (1994).

[26] R. N. Faustov, A. P. Martynenko, Preprint SSU-HEP-97/03, Samara State Univ., Russia (1997), unpublished.

[27] A. Werthmüller, A. Adamczak, R. Jacot-Guillarmod, et al., Hyp. Interact. 116, 1 (1998).

[28] A. Adamczak, D. Bakalov, K. Bakalova, E. Polacco, and C. Rizzo, Hyp. Interact. 136, 1 (2001).

[29] M. P. Faifman, Muon Catal. Fusion 4, 341 (1989); M. Leon, Hyperfine Interact. 82, 151 (1993).

[30] D. Bakalov, M. P. Faifman, L. I. Ponomarev, and S. I. Vinitsky, Nucl. Phys. A 384, 302 (1982).

[31] G. Aissing, H. J. Monkhorst, Phys. Rev. A 42, 3789, (1990).

[32] D. Bakalov, and V. S. Melezhik, Sov. J. Nucl. Phys. 48, 210 (1988)

[33] D. Bakalov, and V. S. Melezhik, JINR Preprint P4-85-952, Dubna (1985) unpublished.

[34] D. Bakalov, K. Bakalova, V. I. Korobov, H. J. Monkhorst, and I. Shimamura, Phys. Rev. A57, 3370 (1998). 